\title{
CONVERGENCE REGIONS FOR THE GENERAL CONTINUED FRACTION
}

\author{
W. J. THRON
}

The purpose of this note is to prove convergence region theorems for continued fractions

$$
\frac{a_{1}}{b_{1}}+\frac{a_{2}}{b_{2}}+\cdots
$$

where the $a_{n}$ and $b_{n}$ are complex numbers. As an application we obtain a new theorem for the associated continued fraction

$$
\frac{a_{1}}{b_{1}+z}+\frac{a_{2}}{b_{2}+z}+\cdots
$$

This continued fraction plays a rôle in the moment problem and is also otherwise of interest as a means of representing analytic functions. For details see Perron [4, chapters 8 and 9$].{ }^{1}$

Recently a continued fraction very similar to (2) has been investigated by Hellinger and Wall [1]. They chose the name " $J$-fraction." A $J$-fraction is a continued fraction of the form

$$
\frac{1}{b_{1}+z}-\frac{c_{1}^{2}}{b_{2}+z}-\frac{c_{2}^{2}}{b_{3}+z}-\cdots .
$$

Our result for $J$-fractions is stated in Theorem $\mathrm{C}$.

The results of this note are closely related to an as yet unpublished work of Wall and Wetzel on "positive definite $J$-fractions." In particular Theorem $\mathrm{C}$ seems to be contained in a theorem of theirs.

In what follows we shall denote by $H(b, \gamma)$ the half-plane (including the boundary) defined by the relation $z \in H(b, \gamma)$ if $\Re\left(z e^{-i \gamma}\right) \geqq b$. For the open half-plane we shall use the notation $H_{0}(b, \gamma)$. It is clear from the context that $b$ is a real number. Further for $a>0, P(a, \gamma)$ shall be the parabolic region (including the boundary) bounded by the curve

$$
\rho \leqq \frac{a^{2} / 2}{1-\cos (\theta-2 \gamma)} .
$$

For $a=0, P(a, \gamma)$ is to be the totality of points $r \cdot e^{i \gamma}, r \geqq 0$. 1943.

Presented to the Society, September 13, 1943; received by the editors April 24,

1 Numbers in brackets refer to the references listed at the end of the paper. 
We are now in a position to state our theorems.

THEOREM A. The continued fraction (1) converges if all the $a_{n}$ lie in a bounded part of the set $P(a, \gamma), a \geqq 0$, and if all $b_{n}$ lie in the halfplane $H(a+\epsilon, \gamma)$, where $\epsilon$ is an arbitrary small positive number.

Even though its statement is somewhat involved it seems worth while to state the following theorem too, as it is a generalization of the parabola theorems ${ }^{2}$ for the continued fractions with $b_{n}=1$.

THEOREM $\mathrm{A}^{\prime}$. In the continued fraction (1) let all $b_{n}$ lie in the angular opening

$$
\left|\arg e^{-i \gamma}\left(b_{n}-1\right)\right|<\pi / 2-\epsilon,
$$

where $-\pi / 2<\gamma<\pi / 2$ and $\epsilon$ is an arbitrary small positive number. Further let all the $a_{n}$ lie in the parabolic region $P(\cos \gamma, \gamma)$. Then the continued fraction (1) converges if and only if $\sum\left|b_{n} d_{n}\right|=\infty$, where $d_{1}=1 / a_{1}$, $d_{n}=1 / d_{n-1} a_{n}$.

It is well known that the last condition of the theorem is satisfied if $\lim \inf a_{n}<\infty$. We note that neither of these two theorems is contained in the other. Both theorems could be used to obtain new "multiple" convergence regions for continued fractions $1+K\left(a_{n} / 1\right)$. For both of these theorems there are corresponding theorems for associated continued fractions and $J$-fractions. We only state the theorems corresponding to Theorem $\mathrm{A}$.

THEOREM B. If real numbers $a \geqq 0, M>0, b$ and $\gamma$ exist such that for all $n \geqq 1, a_{n} \in P(a, \gamma),\left|a_{n}\right|<M$ and $b_{n} \in H(b, \gamma)$ then the associated continued fraction (2) converges uniformly and hence to a holomorphic function of the complex variable $z$ in every closed region contained in the half-plane $H_{0}(a-b, \gamma)$.

It is easily seen that if $a_{n} \in P(a, 0)$ then $c_{n}=a_{n}^{1 / 2}$ satisfies the relation $\left|\Im\left(c_{n}\right)\right| \leqq a / 2$ and conversely. Hence the following theorem for $J$-fractions is an immediate consequence of Theorem B with $\gamma=0$.

THEOREM C. If real numbers $a \geqq 0, M>0$ and $b$ exist such that for all $n \geqq 1,\left|\Im\left(c_{n}\right)\right| \leqq a / 2,\left|c_{n}\right|<M$ and $\Im\left(b_{n}\right) \geqq b$ then the J-fraction (2)' converges uniformly and hence to a holomorphic function of the complex variable $z$ in every closed region contained in the half-plane $\Im(z)>a-b$.

The theorems are based on the following lemma.

LEMma. All the approximants of the continued fraction (1) lie in the half-plane $H(-b / 2, \gamma)$ if for all $n \geqq 1, a_{n} \in P(b, \gamma)$ and $b_{n} \in H(b, \gamma)$, where $b \geqq 0$.

${ }^{2}$ See Leighton and Thron [2] and Paydon and Wall [3]. 
The proof of the lemma is by induction. To perform the induction we require the following two relations: for an arbitrary choice of $a_{n} \in P(b, \gamma), b_{n} \in H(b, \gamma)$ and $v \in H(-b / 2, \gamma)$ the two relations

$$
a_{n} / b_{n} \in H(-b / 2, \gamma)
$$

and

$$
\frac{a_{n}}{b_{n}+v} \in H(-b / 2, \gamma)
$$

must be satisfied. We note that (3) is a consequence of (4) as $v=0 \in H(-b / 2, \gamma)$. Further as $b_{n}$ and $v$ vary independently over their respective regions their sum $b+v$ varies over the half-plane $H(b / 2, \gamma)$. It is then easily seen that relation (4) is satisfied if $P(b, \gamma)$ is the part common to all the regions $c \cdot H(-b / 2, \gamma),(z \in c \cdot H(-b / 2, \gamma)$ if $z=c \cdot v$, where $v \in H(-b / 2, \gamma))$ where $c$ varies over $H(b / 2, \gamma)$. For the proof of this fact we refer the reader to $[2, \S 2]$, where a very similar fact is proved in detail.

We now proceed to the proof of Theorem B. Under the conditions of that theorem $b_{n}+z \in H_{0}(a, \gamma)$ for all $n \geqq 1$. According to the lemma all the approximants of the continued fraction (2) then lie in the halfplane $H(-a / 2, \gamma)$. All approximants are finite. This is seen as follows: In order that an approximant be infinite it is necessary that

$$
-\left(b_{1}+z\right)=\frac{a_{2}}{b_{2}+z}+\cdots+\frac{a_{n}}{b_{n}+z} .
$$

This is impossible as the regions $-H_{0}(a, \gamma)$ and $H(a / 2, \gamma)$ have no point in common.

The approximants of (2) are rational functions of $z$; for $z$ in $H_{0}(a-b, \gamma)$ they are finite and do not take on certain values (more than two), hence by Montel's Theorem the sequence of approximants forms a normal family of holomorphic functions for $z$ in $H_{0}(a-b, \gamma)$.

As the $\left|a_{n}\right|$ are bounded there exists an $M$ such that for $|z|>M$, $z \in H_{0}(a-b, \gamma)$,

$$
\left|b_{n}+z\right|>\left|a_{n}\right|+1
$$

for all $n$. For these values of $z$ the continued fraction converges by Pringsheim's Theorem $[4$, p. 254]. It then follows from the generalized Stieltjes-Vitali Theorem that the continued fraction converges uniformly in every closed region contained in the half-plane $H_{0}(a-b, \gamma)$. This completes the proof of Theorem B.

Theorem A follows from Theorem B if we set $a=b$ and let $z=\epsilon e^{i \gamma}$. 
For the proof of Theorem $\mathrm{A}^{\prime}$ we consider the continued fraction

$$
\frac{a_{1}}{1+d_{1} e^{i \theta_{1} z}}+\frac{a_{2}}{1+d_{2} e^{i \theta_{2} z}}+\cdots,
$$

where $a_{n} \in P(\cos \gamma, \gamma), d_{n} \geqq 0$ and $\left|\theta_{n}-\gamma\right|<\pi / 2-\epsilon$ for all $n \geqq 1$.

Under these conditions the approximants of (5) form a normal family of holomorphic functions for $z$ in the region $D$ defined by

$$
-\delta<\Re(z)<1+\delta,
$$

where $\delta$ is positive and depends on $\gamma$ and $\epsilon$. The proof of this fact is similar to the proof used in the previous case. For $\Re(z)=0$ the continued fraction converges. In this case the partial denominators are real and greater than or equal to 1 and hence (5) can be transformed into a continued fraction of the form

$$
\frac{g_{1}}{1}+\frac{g_{2}}{1}+\cdots
$$

where all $g_{n} \in P(\cos \gamma, \gamma)$. The convergence of this continued fraction follows from the parabola theorems (we are assuming that all conditions of Theorem $\mathrm{A}^{\prime}$ are satisfied). The Stieltjes-Vitali Theorem then insures the convergence of $(5)$ for all $z$ in $D$. If we recall that $\sum\left|c_{n}\right|=\infty$ is a necessary condition for convergence we have Theorem $A^{\prime}$ by setting $z=1$ in (5).

\section{REFERENCES}

1. E. D. Hellinger and H. S. Wall, Contributions to the analytic theory of continued fractions, Ann. of Math. vol. 44 (1943) pp. 103-127.

2. W. Leighton and W. J. Thron, Continued fractions with complex elements, Duke Math. J. vol. 9 (1942) pp. 763-772.

3. J. F. Paydon and H. S. Wall, The continued fraction as a sequence of linear transformations, Duke Math. J. vol. 9 (1942) pp. 360-372.

4. O. Perron, Die Lehre von den Kettenbrilchen, Leipzig, 1929.

The Rice Institute 